
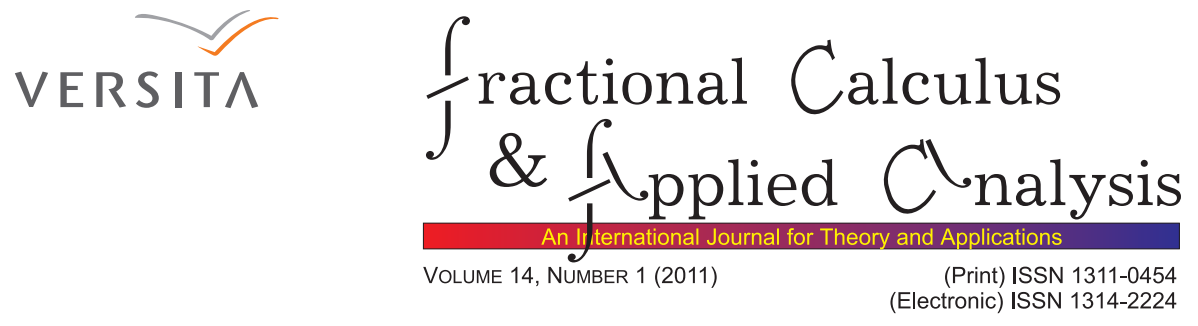

SURVEY PAPER

\title{
INVERSE PROBLEM FOR FRACTIONAL DIFFUSION EQUATION
}

\section{Vu Kim Tuan}

\section{Abstract \\ Dedicated to Prof. Rudolf Gorenflo on his 80th anniversary}

We prove that by taking suitable initial distributions only finitely many measurements on the boundary are required to recover uniquely the diffusion coefficient of a one dimensional fractional diffusion equation. If a lower bound on the diffusion coefficient is known a priori then even only two measurements are sufficient. The technique is based on possibility of extracting the full boundary spectral data from special lateral measurements.

MSC 2010: 26A33, 33E12, 34K29, 34L15, 35K57, 35R30

Key Words and Phrases: fractional diffusion equation, inverse problem, boundary spectral data, eigenfunction expansion

\section{Introduction}

Consider the one-dimensional fractional diffusion equation defined by

$$
\left\{\begin{array}{l}
{ }_{0}^{C} \mathcal{D}_{t}^{\alpha} u(x, t)=u_{x x}(x, t)-q(x) u(x, t), \quad 0<x<\pi, t>0, \\
u_{x}(0, t)-h u(0, t)=0 \\
u_{x}(\pi, t)+H u(\pi, t)=0 \\
u(x, 0)=f(x)
\end{array}\right.
$$

(c) 2011 Diogenes Co., Sofia

pp. 31-55, DOI: 10.2478/s13540-011-0004-x 
where $q \in L_{1}(0, \pi), f \in L_{2}(0, \pi)$, and ${ }_{0}^{C} \mathcal{D}_{t}^{\alpha} u(t), 0<\alpha<1$, is the Caputo fractional derivative, defined by $[9,20$ ]

$$
{ }_{0}^{C} \mathcal{D}_{t}^{\alpha} u(t)=\int_{0}^{t} \frac{(t-x)^{-\alpha}}{\Gamma(1-\alpha)} u^{\prime}(x) d x .
$$

To express the dependence of $u$ on the initial distribution $f$ we sometimes use the notation $u=u^{f}$. The constants $h$ and $H$ in the boundary conditions correspond to the insulation parameters at both ends and are supposed to be known. In a series of papers, see for examples [7, 10, 11, 22], Professor R. Gorenflo and his coauthors have studied fractional diffusion equations and their applications in probability, random walk, and finance. In this paper we are concerned with the inverse problem of (1), namely, the problem of recovery of the diffusion coefficient $q$ from the measurements of lateral distribution $u^{f}(0, t)$ if $h \neq \infty$ (or diffusive flux $u_{x}^{f}(0, t)$ in case $h=\infty$ ) and lateral distribution $u^{f}(\pi, t)$ if $H \neq \infty$ (or diffusive flux $u_{x}^{f}(\pi, t)$ in case $H=\infty)$, on a time interval $\left(T_{0}, T_{1}\right)$, when initial distributions $f$ are given.

For the heat equation (equation (1) with $u_{t}(x, t)$ instead of ${ }_{0}^{C} \mathcal{D}_{t}^{\alpha} u(x, t)$ ) usually the heat coefficient $q$ is recovered from readings of the heat flux $u_{x}(0, t)$ given the temperature $u(0, t)$ at the boundary $x=0$ under zero initial temperature condition $u(x, 0)=0$. In other words, $q$ is determined from infinitely many measurements obtained by the full lateral Dirichlet-toNeumann map, $u(0, t) \rightarrow u_{x}(0, t)$ provided $f(x)=0$. The recovery problem in this setting is over-determined, in the sense that more data is collected than needed, [1, 2, 3, 12, 13]. In [14] the full lateral Dirichlet-to-Robin map yields the boundary spectral data (for the associated Sturm-Liouville problem) that can be used to reconstruct the heat coefficient $q$.

In this paper, instead of giving the full lateral Dirichlet-to-Neumann map, that requires infinitely many measurements, we are given a partial initial-to-boundary map. More precisely, we choose some initial distribution $u(x, 0)=f(x)$ and then measure the lateral diffusions $u^{f}(0, t)$ and $u^{f}(\pi, t)$ if $h$ and $H$ are finite and diffusive fluxes $u_{x}^{f}(0, t)$ and $u_{x}^{f}(\pi, t)$ otherwise. We employ the technique first developed in [4, 5, 6]. The main emphasis in this technique is to provide initial distributions that require finitely many measurements only. The data processing involves extracting boundary spectral data from standard readings of $u(0, t)$ or $u_{x}(0, t)$ at $x=0$ and $u(\pi, t)$ or $u_{x}(\pi, t)$ at $x=\pi$ (Section $\mathbf{5}$ ). Once all boundary spectral data are recovered, Section 6 shows how spectral data, and together with the GelfandLevitan inverse spectral theory, and diffusion coefficient $q$ can be uniquely reconstructed. In Section 7 we show that when the initial distribution is chosen to be a power function $u(x, 0)=x^{\alpha},-\frac{1}{2}<\alpha<0$, then the reading releases almost all boundary spectral data. A blind search will then recover 
all missing boundary spectral data after a finite number of additional measurements (Section 8). We also show how asymptotics of eigenvalues can reveal crucial information about the size of $q$, which then helps us estimate the number of missing eigenvalues (Sections 7, 8). Different assumptions on $q$ lead to algorithms that use a finite number of measurements, at most $N$ measurements, or at most two measurements, to uniquely recover $q$. In fact, we show that for any $q \in L_{1}(0, \pi)$, with a known lower bound, a suitable choice of two initial conditions only $u(x, 0)=f_{i}(x), i=1,2$, a power and a step functions, is enough to recover the diffusion coefficient $q$, (Section 9)

$$
f_{i}(x) \rightarrow\left\{u^{f_{i}}(0, t), u^{f_{i}}(\pi, t)\right\}, t \in\left(T_{0}, T_{1}\right), i=1,2
$$

(with obvious modifications in case $h=\infty$, or $H=\infty$, or both). If instead of a lower bound for $q$ only an upper bound for $\|q\|_{1}$ is known, then we would know explicitly the maximum number of required measurements. The third case would be when no information about $q$ is given, then the blind search will still recover a unique $q$ after a finite number of measurements. However, this number of measurements is known only during the extracting data process.

\section{Preliminaries}

We first look for a series representation of the solution $u$ in (11) which obviously depends on the lateral boundary conditions. Recall that the condition $\varphi^{\prime}(0)-h \varphi(0)=0$ is understood to reduce to $\varphi(0)=0$ if $h=\infty$, and thus, the lateral boundary conditions reduce to mainly four cases for the direct problem, i.e.

$$
\begin{cases}u_{x}(0, t)-h u(0, t)=0, & \text { if } h \neq \infty \\ u(0, t)=0, & \text { if } h=\infty\end{cases}
$$

and

$$
\left\{\begin{array}{ll}
u_{x}(\pi, t)+H u(\pi, t)=0, & \text { if } \quad H \neq \infty \\
u(\pi, t)=0, & \text { if } \quad H=\infty
\end{array} .\right.
$$

For example, the Dirichlet boundary condition $u(0, t)=u(\pi, t)=0$ means $h=H=\infty$. Once $h$ and $H$ have been fixed, denote by $\varphi(x, \lambda)$ the solution of the Sturm-Liouville problem satisfying the initial condition at $x=0$

$$
\left\{\begin{array}{c}
-\varphi^{\prime \prime}(x, \lambda)+q(x) \varphi(x, \lambda)=\lambda \varphi(x, \lambda), \quad 0<x<\pi, \\
\varphi(0, \lambda)=1, \quad \varphi^{\prime}(0, \lambda)=h, \quad \text { if } h \neq \infty, \\
\varphi(0, \lambda)=0, \quad \varphi^{\prime}(0, \lambda)=1, \quad \text { if } h=\infty,
\end{array}\right.
$$

and denote by $\lambda_{n}$ and $\varphi\left(x, \lambda_{n}\right)$ the eigenvalues and associated eigenfunctions of the boundary value problem (3) under the boundary condition

$$
\varphi^{\prime}(\pi, \lambda)+H \varphi(\pi, \lambda)=0, \text { if } \quad H \neq \infty, \quad \varphi(\pi, \lambda)=0, \text { if } \quad H=\infty .
$$


In all cases, $\alpha_{n}$ denotes the $L_{2}(0, \pi)$ norm of $\varphi\left(x, \lambda_{n}\right)$ and $\psi_{n}(x)$ the normalized eigenfunction $\left\|\psi_{n}\right\|_{2}=1$, associated with $\lambda_{n}$

$$
\alpha_{n}=\left\|\varphi\left(x, \lambda_{n}\right)\right\|_{2} \text {, and } \psi_{n}(x)=\frac{1}{\alpha_{n}} \varphi\left(x, \lambda_{n}\right) \text {. }
$$

Thus the generalized Fourier series of any initial condition $f \in L_{2}(0, \pi)$ is given by

$$
f(x)=\sum_{n \geq 0} c_{n} \varphi\left(x, \lambda_{n}\right), \text { where } c_{n}=\frac{\left(f, \psi_{n}\right)}{\alpha_{n}}=\frac{\left(f, \varphi\left(., \lambda_{n}\right)\right)}{\alpha_{n}^{2}} .
$$

We use the separation of variables method to find the solution in the form

$$
u(x, t)=\sum_{n \geq 0} T_{n}(t) \varphi\left(x, \lambda_{n}\right)
$$

then $T_{n}(t)$ is a solution of the fractional differential equation

$$
{ }_{0}^{C} \mathcal{D}_{t}^{\alpha} T_{n}(t)=-\lambda_{n} T_{n}(t), \quad T_{n}(0)=c_{n} .
$$

The solution of this equation can be expressed as follows

$$
T_{n}(t)=c_{n} E_{\alpha}\left(-\lambda_{n} t^{\alpha}\right),
$$

where $E_{\alpha}(x)$ is the Mittag-Leffler function defined by [18, 21]

$$
E_{\alpha}(x)=\sum_{n \geq 0} \frac{x^{n}}{\Gamma(n \alpha+1)} .
$$

So the method of separation of variables yields the solution $u^{f}=u$ of (11) in the series form

$$
u(x, t)=\sum_{n \geq 0} c_{n} E_{\alpha}\left(-\lambda_{n} t^{\alpha}\right) \varphi\left(x, \lambda_{n}\right),
$$

and its spatial derivative in the form

$$
u_{x}(x, t)=\sum_{n \geq 0} c_{n} E_{\alpha}\left(-\lambda_{n} t^{\alpha}\right) \varphi^{\prime}\left(x, \lambda_{n}\right) .
$$

\section{Series representation of the boundary measurements}

Lemma 3.1. For each fixed $t>0$ the series (6) and (7) converge uniformly on $[0, \pi]$.

P r o o f. Let $h \neq \infty$. The asymptotic formulas [15, 17] for the solution $\varphi(x, \lambda)$

its derivative

$$
\varphi(x, \lambda)=\cos \sqrt{\lambda} x+O\left(\frac{1}{\sqrt{\lambda}}\right)
$$




$$
\varphi^{\prime}(x, \lambda)=\sqrt{\lambda} \sin \sqrt{\lambda} x-h \cos \sqrt{\lambda} x+O\left(\frac{1}{\sqrt{\lambda}}\right),
$$

and the eigenvalues

$$
\sqrt{\lambda_{n}}=n+O\left(\frac{1}{n}\right), \quad n \rightarrow \infty
$$

yield

$$
\varphi\left(x, \lambda_{n}\right)=O(1), \quad \varphi^{\prime}\left(x, \lambda_{n}\right)=O(n), \quad x \in[0, \pi] .
$$

Let now $h=\infty$. Then the asymptotic formulas [15, 17] for the solution $\varphi(x, \lambda)$ and its derivative have the form

$$
\begin{aligned}
\varphi(x, \lambda) & =\frac{\sin \sqrt{\lambda} x}{\sqrt{\lambda}}+O\left(\frac{1}{\lambda}\right), \\
\varphi^{\prime}(x, \lambda) & =\cos \sqrt{\lambda} x+O\left(\frac{1}{\sqrt{\lambda}}\right) .
\end{aligned}
$$

For the eigenvalues we have [15, 17]

$$
\begin{aligned}
& \sqrt{\lambda_{n}}=n+\frac{1}{2}+O\left(\frac{1}{n}\right), \quad \text { if } \quad H \neq \infty, \\
& \sqrt{\lambda_{n}}=n+O\left(\frac{1}{n}\right), \quad \text { if } \quad H=\infty .
\end{aligned}
$$

Consequently,

$$
\varphi\left(x, \lambda_{n}\right)=O\left(\frac{1}{n}\right), \quad \varphi^{\prime}\left(x, \lambda_{n}\right)=O(1), \quad x \in[0, \pi] .
$$

The Mittag-Leffler function has the bound ([20])

$$
\left|E_{\alpha}(z)\right|<\frac{C}{1+|z|}, \quad \frac{\alpha \pi}{2}<\mu \leq|\arg (z)| \leq \pi,
$$

hence,

$$
\left|E_{\alpha}\left(-\lambda_{n} t^{\alpha}\right)\right|<\frac{C}{1+\lambda_{n}|t|^{\alpha}}, \quad 0 \leq|\arg (t)| \leq \mu \leq \min \left\{\pi, \frac{\pi}{\alpha}-\frac{\pi}{2}\right\} .
$$

Therefore, for each $t>0$ fixed,

$$
E_{\alpha}\left(-\lambda_{n} t^{\alpha}\right)=O\left(\frac{1}{n^{2}}\right) .
$$

We have now, by the Cauchy-Schwartz inequality, 


$$
\begin{aligned}
& \left|\sum_{n \geq N} c_{n} E_{\alpha}\left(-\lambda_{n} t^{\alpha}\right) \varphi\left(x, \lambda_{n}\right)\right|^{2} \leq \sum_{n \geq N}\left|c_{n}\right|^{2} \sum_{n \geq N}\left|E_{\alpha}\left(-\lambda_{n} t^{\alpha}\right) \varphi\left(x, \lambda_{n}\right)\right|^{2} \\
\leq & C \sum_{n \geq N}\left|c_{n}\right|^{2} \sum_{n \geq N} \frac{1}{n^{4}} \leq \epsilon^{2}, \quad \text { if } \mathrm{N} \text { is chosen large enough, } \quad x \in[0, \pi] .
\end{aligned}
$$

Hence, the series (6) converges uniformly on $[0, \pi]$. Similarly, for the series (77) we have

$$
\begin{aligned}
& \left|\sum_{n \geq N} c_{n} E_{\alpha}\left(-\lambda_{n} t^{\alpha}\right) \varphi^{\prime}\left(x, \lambda_{n}\right)\right|^{2} \leq \sum_{n \geq N}\left|c_{n}\right|^{2} \sum_{n \geq N}\left|E_{\alpha}\left(-\lambda_{n} t^{\alpha}\right) \varphi^{\prime}\left(x, \lambda_{n}\right)\right|^{2} \\
\leq & C \sum_{n \geq N}\left|c_{n}\right|^{2} \sum_{n \geq N} \frac{1}{n^{2}} \leq \epsilon^{2}, \quad \text { if } \mathrm{N} \text { is chosen large enough, } \quad x \in[0, \pi] .
\end{aligned}
$$

Consequently, the series (7) converges uniformly on $[0, \pi]$.

The uniform convergence of the series 6) and (7) on $[0, \pi]$ allows us to represent the readings at the boundary points $x=0$ and $x=\pi$ as series of Mittag-Leffler functions. Possible readings and Mittag-Leffler function series expansions there can be summarized in the following tables.

\begin{tabular}{|l|l|}
\hline$h$ & Measurement at $x=0$ \\
\hline$=\infty$ & $u_{x}(0, t)=\sum_{n \geq 0} c_{n} E_{\alpha}\left(-\lambda_{n} t^{\alpha}\right)$ \\
\hline$\neq \infty$ & $u(0, t)=\sum_{n \geq 0} c_{n} E_{\alpha}\left(-\lambda_{n} t^{\alpha}\right)$ \\
\hline$H$ & Measurement at $x=\pi$ \\
\hline$=\infty$ & $u_{x}(\pi, t)=\sum_{n \geq 0} c_{n} E_{\alpha}\left(-\lambda_{n} t^{\alpha}\right) \varphi^{\prime}\left(\pi, \lambda_{n}\right)$ \\
\hline$\neq \infty$ & $u(\pi, t)=\sum_{n \geq 0} c_{n} E_{\alpha}\left(-\lambda_{n} t^{\alpha}\right) \varphi\left(\pi, \lambda_{n}\right)$ \\
\hline
\end{tabular}




\section{Analyticity of the boundary measurements}

LEMma 4.1. The boundary readings are restrictions on $(0, \infty)$ of functions, analytic in the angle $0 \leq|\arg (t)|<\min \left\{\pi, \frac{\pi}{\alpha}-\frac{\pi}{2}\right\}$.

P r o o f. From (10), (11), (13), and (14) we have, for all four cases $(h \neq \infty, h=\infty, H \neq \infty, H=\infty)$

$$
\varphi\left(\pi, \lambda_{n}\right)=O(1), \quad \varphi^{\prime}\left(\pi, \lambda_{n}\right)=O(n), \quad \lambda_{n} \sim n^{2} .
$$

Let $K$ be the domain $\left\{t, \infty>t_{1} \geq|t| \geq t_{0}>0,0 \leq|\arg (t)| \leq \mu<\right.$ $\left.\min \left\{\pi, \frac{\pi}{\alpha}-\frac{\pi}{2}\right\}\right\}$. The Mittag-Leffler functions $E_{\alpha}\left(-\lambda_{n} t^{\alpha}\right)$ are analytic on $K$. On the compact $K$ from the asymptotic formulas (16) and (19) we have

$$
\begin{aligned}
& \left|\sum_{n \geq N} c_{n} E_{\alpha}\left(-\lambda_{n} t^{\alpha}\right) \varphi^{\prime}\left(\pi, \lambda_{n}\right)\right|^{2} \leq \sum_{n \geq N}\left|c_{n}\right|^{2} \sum_{n \geq N}\left|E_{\alpha}\left(-\lambda_{n} t^{\alpha}\right) \varphi^{\prime}\left(\pi, \lambda_{n}\right)\right|^{2} \\
& \leq C \sum_{n \geq N}\left|c_{n}\right|^{2} \sum_{n \geq N} \frac{n^{2}}{\left(1+\lambda_{n} t_{0}^{\alpha}\right)^{2}} \leq \epsilon^{2}, \quad \text { if } \mathrm{N} \text { is chosen large enough. }
\end{aligned}
$$

Hence, the series

$$
\sum_{n \geq 0} c_{n} E_{\alpha}\left(-\lambda_{n} t^{\alpha}\right) \varphi^{\prime}\left(\pi, \lambda_{n}\right)
$$

converges uniformly on $K$ to an analytic function. Taking $t_{0} \rightarrow 0, t_{1} \rightarrow \infty$, $\mu \rightarrow \min \left\{\pi, \frac{\pi}{\alpha}-\frac{\pi}{2}\right\}$, we arrive at the analyticity of $u_{x}(\pi, t)$ in the stated domain. The proofs are similar for $u(0, t), u_{x}(0, t), u(\pi, t)$.

Lemma 4.1 says that reading $u(0, t)$ on some time interval $t \in\left(T_{0}, T_{1}\right)$ will determine uniquely the boundary values $u(0, t)$ for any $t>0$ by analytic continuation. Similarly for $u_{x}(0, t), u(\pi, t), u_{x}(\pi, t)$.

\section{Extracting boundary spectral data from boundary measurements}

By boundary spectral data (full boundary spectral data) we mean a pair $\left(\lambda_{n}, \varphi\left(\pi, \lambda_{n}\right)\right)$ (the full set $\left.\left\{\left(\lambda_{n}, \varphi\left(\pi, \lambda_{n}\right)\right)\right\}_{n \geq 0}\right)$ if $H \neq \infty$, and a pair $\left(\lambda_{n}, \varphi^{\prime}\left(\pi, \lambda_{n}\right)\right)$ (the full set $\left.\left\{\left(\lambda_{n}, \varphi^{\prime}\left(\pi, \lambda_{n}\right)\right)\right\}_{n \geq 0}\right)$ if $H=\infty$.

Lemma 5.1. Let $c_{n} \neq 0$. Then we can extract the boundary spectral data $\left(\lambda_{n}, \varphi\left(\pi, \lambda_{n}\right)\right.$ ) (for the case $H \neq \infty$ ) or $\left(\lambda_{n}, \varphi^{\prime}\left(\pi, \lambda_{n}\right)\right.$ ) (for the case $H=\infty)$ from the boundary reading on $\left(T_{0}, T_{1}\right)$.

P r o o f. By Lemma 4.1 the functions $u(0, t)(h \neq \infty)$ and $u_{x}(0, t)$ $(h=\infty)$ are analytic in the domain $0 \leq|\arg (t)|<\min \left\{\pi, \frac{\pi}{\alpha}-\frac{\pi}{2}\right\}$. Hence, the reading at $x=0$ on $t \in\left(T_{0}, T_{1}\right)$ determines $u(0, t)(h \neq \infty)$ or $u_{x}(0, t)$ 
$(h=\infty)$ uniquely for $t>0$. Moreover, the reading at $x=0$ has the series representation (18)

$$
g(t)=\sum_{n \geq 0} c_{n} E_{\alpha}\left(-\lambda_{n} t^{\alpha}\right), \quad t>0 .
$$

Recall [18, 20] that the Laplace transform of the Mittag-Leffler function $E_{\alpha}\left(-\lambda_{n} t^{\alpha}\right)$ is $\frac{s^{\alpha-1}}{s^{\alpha}+\lambda_{n}}$

$$
\mathcal{L}\left(E_{\alpha}\left(-\lambda_{n} t^{\alpha}\right)\right)=\int_{0}^{\infty} e^{-s t} E_{\alpha}\left(-\lambda_{n} t^{\alpha}\right) d t=\frac{s^{\alpha-1}}{s^{\alpha}+\lambda_{n}}, \quad \Re(s)>0 .
$$

Since $\lambda_{n} \sim n^{2}$ and $\sum_{n \geq 0}\left|c_{n}\right|^{2}<\infty$, the series

$$
\sum_{n \geq 0}\left|\frac{c_{n} \Re(s)^{\alpha-1}}{\Re(s)^{\alpha}+\lambda_{n}}\right|
$$

is convergent. Therefore, we can interchange the order of integration in the Laplace transform and summation to have

$$
\mathcal{L}(g)(s)=\mathcal{L}\left(\sum_{n \geq 0} c_{n} E_{\alpha}\left(-\lambda_{n} t^{\alpha}\right)\right)=\sum_{n \geq 0} \frac{c_{n} s^{\alpha-1}}{s^{\alpha}+\lambda_{n}} .
$$

Consequently,

$$
s^{1 / \alpha} \mathcal{L}(g)\left(s^{1 / \alpha}\right)=\sum_{n \geq 0} \frac{c_{n} s}{s+\lambda_{n}},
$$

where the series represents an analytic function on $\Re(s)>0$ and is meromorphic over the complex plane with the poles at $\left\{-\lambda_{n}\right\}_{n \geq 0}$ and the corresponding residues $\left\{-c_{n} \lambda_{n}\right\}_{n \geq 0}$. Thus, from $\mathcal{L}(g)(s)$, and therefore, from $g(t), t \in\left(T_{0}, T_{1}\right)$, we can extract $\lambda_{n}$ and $c_{n}$ whenever $c_{n} \neq 0$.

Similarly, if $H \neq \infty$, the reading at $x=\pi$ has the form

$$
g(t)=\sum_{n \geq 0} c_{n} E_{\alpha}\left(-\lambda_{n} t^{\alpha}\right) \varphi\left(\pi, \lambda_{n}\right), \quad t \in\left(T_{0}, T_{1}\right),
$$

then the Laplace transform of $g$ will allow us to determine all $\left\{\lambda_{n}, c_{n} \varphi\left(\pi, \lambda_{n}\right)\right\}$ whenever $c_{n} \neq 0$. Since $c_{n}$ is known from reading at $x=0$, we can extract $\varphi\left(\pi, \lambda_{n}\right)$. And if $H=\infty$, the reading at $x=\pi$ has the form

$$
g(t)=\sum_{n \geq 0} c_{n} E_{\alpha}\left(-\lambda_{n} t^{\alpha}\right) \varphi^{\prime}\left(\pi, \lambda_{n}\right), \quad t \in\left(T_{0}, T_{1}\right)
$$

then the Laplace transform of $g$ will determine all $\left\{\lambda_{n}, c_{n} \varphi^{\prime}\left(\pi, \lambda_{n}\right)\right\}$ whenever $c_{n} \neq 0$. As $c_{n}$ is known from reading at $x=0$, we can extract $\varphi^{\prime}\left(\pi, \lambda_{n}\right)$. Thus we can extract boundary spectral data from lateral readings whenever $c_{n} \neq 0$. 
Lemma 5.1 shows the importance of the condition $c_{n} \neq 0$ in extracting the boundary spectral data from lateral readings. Since

$$
c_{n}=\frac{\left(f, \psi_{n}\right)}{\alpha_{n}},
$$

the condition $c_{n} \neq 0$ is equivalent to

$$
\left(f, \psi_{n}\right) \neq 0
$$

\section{Recovery $q$ from full boundary spectral data}

Before discussing the choice of the initial condition $f$ that guarantees (26) we will show the importance of the full boundary spectral data for recovery of $q$. In [14] it was shown that in case $h=H=\infty$, the full boundary spectral data $\left\{\lambda_{n}, \varphi^{\prime}\left(\pi, \lambda_{n}\right)\right\}_{n \geq 0}$ is enough to determine $q$ uniquely. We now show that the result is also true in the remaining cases which are covered by (2).

Lemma 6.1. The full boundary spectral data $\left\{\lambda_{n}, \varphi\left(\pi, \lambda_{n}\right)\right\}_{n \geq 0}$ when $H \neq \infty$ and $\left\{\lambda_{n}, \varphi^{\prime}\left(\pi, \lambda_{n}\right)\right\}_{n \geq 0}$ when $H=\infty$ determine $q$ uniquely.

$\mathrm{P}$ r o o f. The proof makes use of the analytic property of the solution $\varphi(x, \lambda)$ of (3) in the variable $\lambda$. The eigenvalues $\lambda_{n}$ of (3) are the zeros of $\varphi^{\prime}(\pi, \lambda)+H \varphi(\pi, \lambda)$, if $H \neq \infty$, or the zeros of $\varphi(\pi, \lambda)$, if $H=\infty$. Conversely, the entire function

$$
\Psi(\lambda):= \begin{cases}\varphi^{\prime}(\pi, \lambda)+H \varphi(\pi, \lambda), & H \neq \infty \\ \varphi(\pi, \lambda), & H=\infty\end{cases}
$$

is determined uniquely by its zeros $\left\{\lambda_{n}\right\}_{n \geq 0}$ [19]

$$
\Psi(\lambda)=\pi\left(\lambda_{0}-\lambda\right) \prod_{n \geq 1}\left(\frac{\lambda_{n}-\lambda}{n^{2}}\right) .
$$

Hence, $\Psi(\lambda)$ and $\partial_{\lambda} \Psi(\lambda)$ are uniquely determined if $\left\{\lambda_{n}\right\}_{n \geq 0}$ are known. We now prove that $\Psi(\lambda)$ and $\left\{\varphi\left(\pi, \lambda_{n}\right)\right\}_{n \geq 0}$ (or $\left\{\varphi^{\prime}\left(\pi, \lambda_{n}\right)\right\}_{n \geq 0}$ if $\left.H=\infty\right)$ are enough to evaluate the norms $\alpha_{n}$. In fact,

$$
\varphi^{2}=\left(\varphi^{\prime} \partial_{\lambda} \varphi-\varphi \partial_{\lambda} \varphi^{\prime}\right)^{\prime}
$$

Therefore, 
- If $H \neq \infty$, then

$$
\begin{aligned}
\alpha_{n}^{2}=\int_{0}^{\pi} \varphi^{2}\left(x, \lambda_{n}\right) d x & =\varphi^{\prime}\left(\pi, \lambda_{n}\right) \partial_{\lambda} \varphi\left(\pi, \lambda_{n}\right)-\varphi\left(\pi, \lambda_{n}\right) \partial_{\lambda} \varphi^{\prime}\left(\pi, \lambda_{n}\right) \\
& -\varphi^{\prime}\left(0, \lambda_{n}\right) \partial_{\lambda} \varphi\left(0, \lambda_{n}\right)+\varphi\left(0, \lambda_{n}\right) \partial_{\lambda} \varphi^{\prime}\left(0, \lambda_{n}\right) \\
& =\varphi^{\prime}\left(\pi, \lambda_{n}\right) \partial_{\lambda} \varphi\left(\pi, \lambda_{n}\right)-\varphi\left(\pi, \lambda_{n}\right) \partial_{\lambda} \varphi^{\prime}\left(\pi, \lambda_{n}\right) \\
& =-\varphi\left(\pi, \lambda_{n}\right) \partial_{\lambda} \Psi\left(\lambda_{n}\right) .
\end{aligned}
$$

Since $\partial_{\lambda} \Psi\left(\lambda_{n}\right)$ and $\varphi\left(\pi, \lambda_{n}\right)$ are known, all norming constants $\alpha_{n}$ can be determined.

- In case $H=\infty$, we have $\varphi\left(\pi, \lambda_{n}\right)=0$, and therefore,

$$
\begin{aligned}
\alpha_{n}^{2} & =\varphi^{\prime}\left(\pi, \lambda_{n}\right) \partial_{\lambda} \varphi\left(\pi, \lambda_{n}\right)-\varphi\left(\pi, \lambda_{n}\right) \partial_{\lambda} \varphi^{\prime}\left(\pi, \lambda_{n}\right) \\
& =\varphi^{\prime}\left(\pi, \lambda_{n}\right) \partial_{\lambda} \Psi\left(\lambda_{n}\right) .
\end{aligned}
$$

Hence, all norming constants $\alpha_{n}$ can be defined from the full boundary spectral data $\left\{\lambda_{n}, \varphi^{\prime}\left(\pi, \lambda_{n}\right)\right\}_{n \geq 0}$.

Recall that if we know the complete spectral data $\left\{\lambda_{n}, \alpha_{n}\right\}_{n \geq 0}$, then we can recover potential $q$ uniquely, a well known result of the GelfandLevitan-Gasymov theory [15, 17]. Indeed, the complete spectral data define the spectral function for the Sturm-Liouville problem (3) and (4)

$$
\Gamma(\lambda)=\sum_{\lambda_{n} \leq \lambda} \alpha_{n}
$$

Let

$$
F(x, t)=\int_{-\infty}^{\infty} \cos (x \sqrt{\lambda}) \cos (t \sqrt{\lambda}) d\left(\Gamma(\lambda)-\frac{2}{\pi} \sqrt{\lambda_{+}}\right),
$$

where $\lambda_{+}=\lambda$ if $\lambda>0$, and $\lambda_{+}=0$ otherwise. Solving the Fredholm integral equation

$$
F(x, t)+K(x, t)+\int_{0}^{x} K(x, s) F(s, t) d s=0, \quad 0 \leq t \leq x \leq \pi,
$$

for $K(x, t)$, which is known to be differentiable, the function $q$ is then recovered by $q(x)=\frac{1}{2} \frac{d}{d x} K(x, x)$. Hence, the knowledge of the full boundary spectral data $\left\{\lambda_{n}, \varphi\left(\pi, \lambda_{n}\right)\right\}_{n \geq 0}($ case $H \neq \infty)$, or $\left\{\lambda_{n}, \varphi^{\prime}\left(\pi, \lambda_{n}\right)\right\}_{n \geq 0}$ (case $\left.H=\infty\right)$, determines $q$ uniquely.

Thus the main issue now at hand is how to choose initial conditions $u(x, 0)=f(x)$ so that we can extract a complete set of boundary spectral data from only a finite number of measurements (18). From here on we follow the technique developed in [4, 5, with some proofs and estimates being directly borrowed from [4]. 


\section{Recovering large eigenvalues}

Now that we know how to extract the eigenvalues $\lambda_{n}$ from the measurement $u^{f}(0, t)$, (or $\left.u_{x}^{f}(0, t)\right)$, we face the question of their completeness. Thus given any $f \in L_{2}(0, \pi)$, we have $f(x)=\sum_{n>0} c_{n} \varphi\left(x, \lambda_{n}\right)$, and to retrieve all eigenvalues $\lambda_{n}$ from (20) we simply need to ensure that $c_{n} \neq 0$ for all $n \geq 0$. The main difficulty here is that since the orthogonal system $\varphi\left(x, \lambda_{n}\right)$ is unknown, the condition $c_{n} \neq 0$ is not easily verified. The key idea in [4] is to use a special initial condition to ensure that its eigenfunction expansion contains nearly all eigenfunctions. We start with the following

Lemma 7.1. Let $f(x)=x^{\alpha}, \alpha \in(-1 / 2,0)$, then there is $N>0$ such that $c_{n} \neq 0$ for $n \geq N$.

P r o o f. The condition $\alpha \in(-1 / 2,0)$ guarantees that $f \in L_{2}(0, \pi)$, therefore its eigenfunction expansion converges in the mean. We distinguish three cases:

a) If $h \neq \infty, H \neq \infty$, then the normalized eigenfunction $\psi_{n}$ has the following asymptotic expansion ([17])

$$
\psi_{n}(x)=\sqrt{\frac{2}{\pi}} \cos n x+O\left(\frac{1}{n}\right), \quad n \rightarrow \infty .
$$

Hence for $f(x)=x^{\alpha}$ we have

$$
\int_{0}^{\pi} f(x) \psi_{n}(x) d x=n^{-\alpha-1} \sqrt{\frac{2}{\pi}} \int_{0}^{n \pi} \xi^{\alpha} \cos \xi d \xi+O\left(\frac{1}{n}\right) .
$$

As $n \rightarrow \infty$, the last integral converges to a nonzero limit

$$
c(\alpha):=\lim _{n \rightarrow \infty} \sqrt{\frac{2}{\pi}} \int_{0}^{n \pi} \xi^{\alpha} \cos \xi d \xi=\sqrt{\frac{2}{\pi}} \int_{0}^{\infty} \xi^{\alpha} \cos \xi d \xi=2^{\alpha+1 / 2} \frac{\Gamma\left(\frac{\alpha+1}{2}\right)}{\Gamma\left(-\frac{\alpha}{2}\right)},
$$

and so

$$
\int_{0}^{\pi} f(x) \psi_{n}(x) d x=\frac{c(\alpha)}{n^{\alpha+1}}+o\left(\frac{1}{n^{\alpha+1}}\right) .
$$

Therefore, from (5) we have

$$
\alpha_{n} c_{n} n^{\alpha+1} \rightarrow c(\alpha) \neq 0, \quad \text { as } \quad n \rightarrow \infty .
$$

Thus, the choice $f(x)=x^{\alpha},-1 / 2<\alpha<0$, guarantees the existence of $N$ such that $c_{n} \neq 0$ for $n \geq N$. 
b) Let $h=\infty$, but $H \neq \infty$ (the case $h \neq \infty, H=\infty$ is similar). Then ([17])

$$
\psi_{n}(x)=\sqrt{\frac{2}{\pi}} \sin \left(n+\frac{1}{2}\right) x+O\left(\frac{1}{n}\right), \quad n \rightarrow \infty,
$$

and therefore

$$
\alpha_{n} c_{n}=\int_{0}^{\pi} x^{\alpha} \psi_{n}(x) d x=\frac{\widetilde{c}(\alpha)}{\left(n+\frac{1}{2}\right)^{\alpha+1}}+o\left(\frac{1}{n^{\alpha+1}}\right),
$$

where

$$
\widetilde{c}(\alpha):=\sqrt{\frac{2}{\pi}} \int_{0}^{\infty} \xi^{\alpha} \sin \xi d \xi=\sqrt{\frac{2}{\pi}} \Gamma(\alpha+1) \sin \frac{(\alpha+1) \pi}{2} \neq 0 .
$$

Consequently, there exists $N$ such that $c_{n} \neq 0$ for $n \geq N$.

c) Finally, let $h=H=\infty$. Then it follows again from [17]

$$
\psi_{n}(x)=\sqrt{\frac{2}{\pi}} \sin n x+O\left(\frac{1}{n}\right), \quad n \rightarrow \infty,
$$

that

$$
\alpha_{n} c_{n}=\int_{0}^{\pi} x^{\alpha} \psi_{n}(x) d x=\frac{\widetilde{c}(\alpha)}{n^{\alpha+1}}+o\left(\frac{1}{n^{\alpha+1}}\right) .
$$

where $\widetilde{c}(\alpha)$ is defined as in (32). Hence, there exists $N$ such that $c_{n} \neq 0$ for $n \geq N$.

Observe that in order to estimate $N$ we need to find a bound on $o\left(\frac{1}{n^{\alpha+1}}\right)$. If no extra condition is imposed on $q$ then the actual value $N$ cannot be estimated. Let us point out a very important case when $N$ can be determined. Assume the $L_{1}$-norm of $q$ is known a priori to be bounded by $Q$, the actual value $N$ then can be found through the following

Lemma 7.2. Let $\|q\|_{1} \leq Q$ and $f(x)=x^{\alpha}, \alpha \in(-1 / 2,0)$. Then $c_{n} \neq 0$ for $\lambda_{n} \geq N$, where

$$
N=\max \left\{4 Q^{2}, \frac{c(\alpha)^{\frac{2}{\alpha}}}{2^{\frac{3}{\alpha}} \pi^{2-\frac{1}{\alpha}}}\left[1+\frac{\pi}{\alpha+1}(Q+h)\right]^{\frac{-2}{\alpha}}\right\},
$$

in case $h \neq \infty$, and

$$
N=\max \left\{4 Q^{2}, \frac{(\widetilde{c}(\alpha))^{\frac{2}{\alpha}}}{2^{\frac{3}{\alpha}} \pi^{2-\frac{1}{\alpha}}}\left[1+\frac{\pi}{\alpha+1} Q\right]^{\frac{-2}{\alpha}}\right\},
$$

in case $h=\infty$. Here $c(\alpha)$ is given by (31), and $\widetilde{c}(\alpha)$ by (32). 
P r o o f. We distinguish two cases:

a) Let $h \neq \infty$. From the equation [17]

$$
\varphi(x, \lambda)=\cos \sqrt{\lambda} x+\frac{h}{\sqrt{\lambda}} \sin \sqrt{\lambda} x+\frac{1}{\sqrt{\lambda}} \int_{0}^{x} \sin (\sqrt{\lambda}(x-t)) q(t) \varphi(t, \lambda) d t,
$$

we have

$$
\|\varphi(., \lambda)\|_{\infty} \leq 1+\frac{h}{\sqrt{\lambda}}+\frac{\|\varphi(., \lambda)\|_{\infty}\|q\|_{1}}{\sqrt{\lambda}} .
$$

Therefore, when $\sqrt{\lambda}>\|q\|_{1}$ we obtain

$$
\|\varphi(., \lambda)\|_{\infty} \leq \frac{1+\frac{h}{\sqrt{\lambda}}}{1-\frac{\|q\|_{1}}{\sqrt{\lambda}}} .
$$

Consequently, (35) and (36) yield

$$
\begin{aligned}
& \|\varphi(x, \lambda)-\cos \sqrt{\lambda} x\|_{\infty} \leq \frac{h}{\sqrt{\lambda}}+\frac{\|\varphi(., \lambda)\|_{\infty}\|q\|_{1}}{\sqrt{\lambda}} \\
& \leq \frac{1}{\sqrt{\lambda}}\left[h+\frac{1+\frac{h}{\sqrt{\lambda}}}{1-\frac{\|q\|_{1}}{\sqrt{\lambda}}}\|q\|_{1}\right]=\frac{1}{\sqrt{\lambda}} \frac{\|q\|_{1}+h}{1-\frac{\|q\|_{1}}{\sqrt{\lambda}}}
\end{aligned}
$$

We have

$$
\begin{array}{r}
\left|\int_{0}^{\pi} x^{\alpha} \varphi\left(x, \lambda_{n}\right) d x-\int_{0}^{\pi} x^{\alpha} \cos \sqrt{\lambda_{n}} x d x\right| \\
\leq \frac{1}{\sqrt{\lambda_{n}}} \frac{\|q\|_{1}+\frac{h}{\sqrt{\lambda_{n}}}}{1-\frac{\|q\|_{1}}{\sqrt{\lambda_{n}}}} \int_{0}^{\pi} x^{\alpha} d x=\frac{1}{\sqrt{\lambda_{n}}} \frac{\|q\|_{1}+h}{1-\frac{\|q\|_{1}}{\sqrt{\lambda_{n}}}} \frac{\pi^{\alpha+1}}{\alpha+1} .
\end{array}
$$

Since $x^{\alpha}$ is decreasing, the use of the second mean value theorem

$$
\left|\int_{\pi \sqrt{\lambda_{n}}}^{\infty} x^{\alpha} \cos x d x\right|=\left|\left(\pi \sqrt{\lambda_{n}}\right)^{\alpha} \int_{\pi \sqrt{\lambda_{n}}}^{c} \cos x d x\right| \leq 2\left(\pi \sqrt{\lambda_{n}}\right)^{\alpha}
$$

yields the estimate

$$
\begin{array}{r}
\left|\int_{0}^{\pi} x^{\alpha} \cos \sqrt{\lambda_{n}} x d x-\frac{\sqrt{\pi} c(\alpha)}{\sqrt{2} \lambda_{n}^{\frac{\alpha+1}{2}}}\right| \\
=\left|\frac{1}{\lambda_{n}^{\frac{\alpha+1}{2}}} \int_{0}^{\pi \sqrt{\lambda_{n}}} t^{\alpha} \cos t d t-\frac{1}{\lambda_{n}^{\frac{\alpha+1}{2}}} \int_{0}^{\infty} t^{\alpha} \cos t d t\right| \\
=\left|\frac{1}{\lambda_{n}^{\frac{\alpha+1}{2}}} \int_{\pi \sqrt{\lambda_{n}}}^{\infty} t^{\alpha} \cos t d t\right| \leq \frac{2 \pi^{\alpha}}{\sqrt{\lambda_{n}}} .
\end{array}
$$


Combining (37) and (38), we arrive at

$$
\left|\alpha_{n}^{2} c_{n}-\frac{\sqrt{\pi} c(\alpha)}{\sqrt{2} \lambda_{n}^{\frac{\alpha+1}{2}}}\right| \leq \frac{\pi^{\alpha}}{\sqrt{\lambda_{n}}}\left[\frac{\|q\|_{1}+h}{1-\frac{\|q\|_{1}}{\sqrt{\lambda_{n}}}} \frac{\pi}{\alpha+1}+2\right] .
$$

Since $c(\alpha)>0$, from (31) we derive that $c_{n} \neq 0$ if

$$
\begin{aligned}
\frac{\sqrt{\pi} c(\alpha)}{\sqrt{2} \lambda_{n}^{\frac{\alpha+1}{2}}} & >\frac{\pi^{\alpha}}{\sqrt{\lambda_{n}}}\left(\frac{\|q\|_{1}+h}{1-\frac{\|q\|_{1}}{\sqrt{\lambda_{n}}}} \frac{\pi}{\alpha+1}+2\right), \\
\lambda_{n}^{-\frac{\alpha}{2}} & >\frac{\sqrt{2} \pi^{\alpha-\frac{1}{2}}}{c(\alpha)}\left(\frac{\|q\|_{1}+h}{1-\frac{\|q\|_{1}}{\sqrt{\lambda_{n}}}} \frac{\pi}{\alpha+1}+2\right) .
\end{aligned}
$$

It holds when

$$
\lambda_{n}>\max \left\{4\|q\|_{1}^{2}, \frac{c^{\frac{2}{\alpha}}(\alpha)}{2^{\frac{3}{\alpha}} \pi^{2-\frac{1}{\alpha}}}\left(1+\frac{\pi}{\alpha+1}\left(\|q\|_{1}+h\right)\right)^{-\frac{2}{\alpha}}\right\} .
$$

b) The second case $h=\infty$ is similar since the variation of parameters yields ([17])

$$
\varphi(x, \lambda)=\frac{\sin \sqrt{\lambda} x}{\sqrt{\lambda}}+\frac{1}{\sqrt{\lambda}} \int_{0}^{x} \sin \sqrt{\lambda}(x-t) q(t) \varphi(t, \lambda) d t .
$$

Therefore,

$$
\|\varphi(., \lambda)\|_{\infty} \leq \frac{1}{\sqrt{\lambda}}+\|\varphi(., \lambda)\|_{\infty}\|q\|_{1} \frac{1}{\sqrt{\lambda}} .
$$

Thus, when $\sqrt{\lambda}>\|q\|_{1}$ we get

$$
\|\varphi(., \lambda)\|_{\infty} \leq \frac{1}{\sqrt{\lambda}\left(1-\frac{\|q\|_{1}}{\sqrt{\lambda}}\right)}
$$

and

$$
\begin{aligned}
& \left\|\varphi(x, \lambda)-\frac{\sin \sqrt{\lambda} x}{\sqrt{\lambda}}\right\|_{\infty} \leq \frac{\|\varphi(., \lambda)\|_{\infty}\|q\|_{1}}{\sqrt{\lambda}} \\
& \leq \frac{1}{\sqrt{\lambda}} \frac{1}{\sqrt{\lambda}\left(1-\frac{\|q\|_{1}}{\sqrt{\lambda}}\right)}\|q\|_{1}=\frac{1}{\lambda} \frac{\|q\|_{1}}{1-\frac{\|q\|_{1}}{\sqrt{\lambda}}} .
\end{aligned}
$$

Consequently, 


$$
\begin{aligned}
& \left|\int_{0}^{\pi} x^{\alpha} \varphi\left(x, \lambda_{n}\right) d x-\int_{0}^{\pi} x^{\alpha} \frac{\sin \sqrt{\lambda} x}{\sqrt{\lambda}} d x\right| \\
\leq & \frac{1}{\lambda} \frac{\|q\|_{1}}{1-\frac{\|q\|_{1}}{\sqrt{\lambda}}} \int_{0}^{\pi} x^{\alpha} d x=\frac{1}{\lambda} \frac{\|q\|_{1}}{1-\frac{\|q\|_{1}}{\sqrt{\lambda}}} \frac{\pi^{\alpha+1}}{\alpha+1} .
\end{aligned}
$$

Since $x^{\alpha}$ is decreasing, the second mean value theorem gives

$$
\left|\int_{\pi \sqrt{\lambda_{n}}}^{\infty} x^{\alpha} \sin x d x\right|=\left|\left(\pi \sqrt{\lambda_{n}}\right)^{\alpha} \int_{\pi \sqrt{\lambda_{n}}}^{c} \sin x d x\right| \leq 2\left(\pi \sqrt{\lambda_{n}}\right)^{\alpha} .
$$

Hence,

$$
\begin{array}{r}
\left|\int_{0}^{\pi} x^{\alpha} \frac{\sin \sqrt{\lambda} x}{\sqrt{\lambda}} d x-\frac{\sqrt{\pi} \widetilde{c}(\alpha)}{\sqrt{2} \lambda_{n}^{\frac{\alpha}{2}+1}}\right| \\
=\left|\frac{1}{\lambda_{n}^{\frac{\alpha}{2}+1}} \int_{0}^{\pi \sqrt{\lambda_{n}}} t^{\alpha} \sin t d t-\frac{1}{\lambda_{n}^{\frac{\alpha}{2}}+1} \int_{0}^{\infty} t^{\alpha} \sin t d t\right| \\
=\left|\frac{1}{\lambda_{n}^{\frac{\alpha}{2}+1}} \int_{\pi \sqrt{\lambda_{n}}}^{\infty} t^{\alpha} \sin t d t\right| \leq \frac{2 \pi^{\alpha}}{\lambda_{n}} .
\end{array}
$$

Consequently,

$$
\left|\alpha_{n}^{2} c_{n}-\frac{\sqrt{\pi} \widetilde{c}(\alpha)}{\sqrt{2} \lambda_{n}^{\frac{\alpha}{2}+1}}\right| \leq \frac{1}{\lambda_{n}}\left[\frac{\|q\|_{1}}{1-\frac{\|q\|_{1}}{\sqrt{\lambda}}} \frac{\pi^{\alpha+1}}{\alpha+1}+2 \pi^{\alpha}\right] .
$$

Since $\widetilde{c}(\alpha)>0$, then $c_{n} \neq 0$ if

$$
\frac{\sqrt{\pi} \widetilde{c}(\alpha)}{\sqrt{2} \lambda_{n}^{\frac{\alpha}{2}+1}}>\frac{1}{\lambda_{n}}\left[\frac{\|q\|_{1}}{1-\frac{\|q\|_{1}}{\sqrt{\lambda}}} \frac{\pi^{\alpha+1}}{\alpha+1}+2 \pi^{\alpha}\right] .
$$

In other words, $c_{n} \neq 0$ when

$$
\lambda_{n}>\max \left\{4\|q\|_{1}^{2}, \frac{(\widetilde{c}(\alpha))^{\frac{2}{\alpha}}}{2^{\frac{3}{\alpha}} \pi^{2-\frac{1}{\alpha}}}\left[1+\frac{\pi\|q\|_{1}}{\alpha+1}\right]^{-\frac{2}{\alpha}}\right\} .
$$

Lemma 7.2 shows how crucial is the upper bound $Q$ of $\|q\|_{1}$ for estimating the number of possible missing eigenvalues. Observe that if $q \geq 0$, then $\|q\|_{1}=\int_{0}^{\pi} q(x) d x$, a constant that appears in the asymptotics of the 
eigenvalues ([17]):

$$
\begin{aligned}
\sqrt{\lambda_{n}} & =n+a_{0}+\frac{a_{1}+a_{2}}{n}+o\left(\frac{1}{n}\right), \\
a_{1} & =\frac{1}{2 \pi} \int_{0}^{\pi} q(x) d x \\
a_{0} & = \begin{cases}\frac{1}{2} \text { if } h=\infty, H \neq \infty, \text { or } h \neq \infty, H=\infty \\
0 \quad \text { otherwise }\end{cases} \\
a_{2} & =\left\{\begin{array}{lll}
0 & \text { if } h=\infty, H=\infty \\
\frac{h+H}{\pi} & \text { if } h \neq \infty, H \neq \infty \\
\frac{H^{\pi}}{\pi} & \text { if } \quad h=\infty, H \neq \infty \\
\frac{h}{\pi} & \text { if } \quad h \neq \infty, H=\infty
\end{array} .\right.
\end{aligned}
$$

Thus obviously, if it is a priori known that $q \geq 0$, then $\|q\|_{1}$ can be computed from the recovered eigenvalues.

Lemma 7.3. Given that $q \geq 0$ then

$$
\|q\|_{1}=2 \pi \lim _{n \rightarrow \infty}\left[\left(\sqrt{\lambda_{n}}-n-a_{0}\right) n-a_{2}\right] .
$$

P r o o f. Follows from (39).

The case $q(x) \geq-C$ can also be reduced to the above case. Consider the translated problem

$$
-y^{\prime \prime}(x)+\widetilde{q}(x) y(x)=\widetilde{\lambda} y(x),
$$

where $\widetilde{q}:=q+C \geq 0, \widetilde{\lambda}:=\lambda+C$, and the same boundary conditions as in (3) and (4). Thus we would recover $\widetilde{q}$ first and then $q$ by subtracting $C$. Since $\|q(x)+C\|_{1}=2 \pi \lim _{n \rightarrow \infty}\left[\left(\sqrt{C+\lambda_{n}}-n-a_{0}\right) n-a_{2}\right]$, and $\|q(x)\|_{1} \leq$ $\|q(x)+C\|_{1}+\|C\|_{1}=\|q(x)+C\|_{1}+\pi C$, we have the following

Corollary 7.1. Given $q \geq-C$, then

$$
\|q(x)\|_{1} \leq Q:=\pi C+2 \pi \lim _{n \rightarrow \infty}\left[\left(\sqrt{C+\lambda_{n}}-n-a_{0}\right) n-a_{2}\right] .
$$

\section{Recovering low eigenvalues}

The previous section guarantees that with the initial distribution $f(x)=$ $x^{\alpha}$ we can extract all large eigenvalues from lateral reading. But some low eigenvalues may go missing in the first reading. We now need to find out the number of missing eigenvalues and then recover them by measuring lateral data from additional initial conditions. Because $N$ is not known explicitly, 
to proceed further we use the method of false position. Let $M$ be the number starting from which all recovered eigenvalues larger than $(M-1 / 2)^{2}$ fall in intervals $\left(\left(k+a_{0}-1 / 2\right)^{2},\left(k+a_{0}+1 / 2\right)^{2}\right)$, where $k \geq M$, and $a_{0}$ is defined in (39). More importantly, each of these intervals contains exactly one recovered eigenvalue. The existence of such an $M$ comes from (39) and Lemma 7.1 which says that after a certain $N$ no eigenvalue is missing. Therefore we can tentatively rank, maybe falsely, the eigenvalue contained in $\left(\left(k+a_{0}-1 / 2\right)^{2},\left(k+a_{0}+1 / 2\right)^{2}\right), k \geq M$, as $\lambda_{k}$ and this ranking is certainly true for $k \geq P:=\max (M, N)$.

Now suppose there are only $L$ eigenvalues recovered in $\left(-\infty, \lambda_{M}\right)$ after the first reading. Since $\left[\lambda_{M}, \lambda_{P}\right)$ contains $P-M$ eigenvalues $\left\{\lambda_{M}, \ldots, \lambda_{P-1}\right\}$, the actual number of recovered eigenvalues in $\left(-\infty, \lambda_{P}\right)$ is $L+(P-M)$. Recall that the ranking holds true from $\lambda_{P}$ and up and therefore there must be $P$ eigenvalues before $\lambda_{P}$. Hence, only $M-L$ eigenvalues are missing in the first reading, and so by knowing $M$, we readily know the number of missing eigenvalues.

For example, starting for $k \geq 50$, each of the intervals $\left(\left(k+a_{0}-\right.\right.$ $\left.1 / 2)^{2},\left(k+a_{0}+1 / 2\right)^{2}\right)$ contains exactly one recovered eigenvalue, which can be ranked tentatively as $\lambda_{k}$, and none falls between them. If by counting, we have found only 40 eigenvalues less than $\lambda_{50}$, it means that we miss exactly 10 eigenvalues after the first reading. The difficulty in ranking here is that the missing eigenvalues are not necessarily below $\lambda_{50}$. That is why it is important to first recover all the missing eigenvalues and only after that can the ranking be finalized. The method to search and rank these missing eigenvalues is close in spirit to method of false position as the first assumption on ranking may be incorrect.

We now propose a way to dig out all the missing eigenvalues in $\left\{\lambda_{0}, \ldots\right.$, $\left.\lambda_{P-1}\right\}$ by choosing appropriate initial conditions to ensure that the missing eigenvalues are necessarily embedded in the response of $u^{f}(0, t)$ (or $u_{x}^{f}(0, t)$, if $h=\infty)$. In other words we need to find initial conditions that would guarantee $c_{n} \neq 0$ for $n=0, \ldots, P-1$. We start off with the following observation.

Lemma 8.1. For any fixed $j$ the coefficients $c_{j}(k)=\int_{0}^{\pi} x^{k} \varphi\left(x, \lambda_{j}\right) d x$ $\neq 0$ for at least one $k=0, \ldots, j$.

P r o o f. Consider the case $h \neq \infty$. The case $h=\infty$ is quite similar. Let us assume that

$$
c_{j}(k)=0 \text { for } \quad \text { all } \quad k=0, \ldots, j \text {. }
$$


Recall that the $j^{\text {th }}$ eigenfunction of (3) has $j$ simple zeroes [17], say $a_{j k}$, and so $\varphi\left(a_{j k}, \lambda_{j}\right)=0$ for $k=1, \ldots, j$. Given the zeros $a_{j k}$ we can construct the polynomial

$$
P_{j}(x)=\varepsilon_{j} \prod_{k=1}^{j}\left(x-a_{j k}\right)=\sum_{k=0}^{j} b_{k} x^{k}
$$

where $\varepsilon_{j}$ is chosen so that $P(0)=1$. Since $P_{j}$ and $\varphi\left(., \lambda_{j}\right)$ have the same degree, the same zeros, and both satisfy $P_{j}(0)=\varphi\left(0, \lambda_{j}\right)=1$, they must have the same sign for all $x \in[0, \pi]$, that is

$$
P_{j}(x) \varphi\left(x, \lambda_{j}\right)>0 \text { if } \quad x \neq a_{j k}, \quad k=1, \cdots, j .
$$

Therefore we conclude that

$$
\int_{0}^{\pi} P_{j}(x) \varphi\left(x, \lambda_{j}\right) d x>0
$$

and at the same time by (40) and (41) we have

$$
\int_{0}^{\pi} P_{j}(x) \varphi\left(x, \lambda_{j}\right) d x=\sum_{k=0}^{j} b_{k} \int_{0}^{\pi} x^{k} \varphi\left(x, \lambda_{j}\right) d x=\sum_{k=0}^{j} b_{k} c_{j}(k)=0,
$$

which contradicts (42).

The above result says that the family $\left\{u^{x^{k}}(0, t)\right\}_{k=0}^{P-1}$ will deliver the first $P$ eigenvalues, but unfortunately the number $P$ is unknown. Nevertheless, we can still use the knowledge of the number of missing eigenvalues, i.e. $M-L$, as a stopping rule.

Thus we start by reading $u^{x^{0}}(0, t)$ (or $u_{x}^{x^{0}}(0, t)$ if $h=\infty$ ) to find missing eigenvalues. If after that eigenvalues are still missing, we go to the next $u^{x^{1}}(0, t)$, and so on until we have recovered all $M-L$ missing eigenvalues. Lemma 8.1 guarantees that this process stops at some point (at most after looking at $\left.u^{x^{P-1}}(0, t)\right)$ and so the number of measurements is finite. After that, we can finally rearrange and update the tentative ranking.

\section{The second initial condition}

When a lower bound of $q$ is known, it turns out that only one more special reading is necessary to unravel all missing eigenvalues.

Lemma 9.1. Let $q(x) \geq-C$, where $C$ is a nonnegative number, and

$$
\chi_{\nu}(x)=\left\{\begin{array}{ccc}
1 & \text { if } & 0<x<\nu \\
0 & \text { if } & \nu \leq x<\pi
\end{array},\right.
$$

where

$$
\nu=\frac{\pi}{4 \sqrt{\lambda_{n}+C}} .
$$


Then $c_{j}=\left(\chi_{\nu}, \varphi_{j}\right) \neq 0$ for $j=0, \cdots, n$.

P r o o f. Compare two initial value problems

$$
\left\{\begin{array}{l}
-\varphi^{\prime \prime}\left(x, \lambda_{n}\right)+q(x) \varphi\left(x, \lambda_{n}\right)=\lambda_{n} \varphi\left(x, \lambda_{n}\right) \\
\varphi\left(0, \lambda_{n}\right)=1, \quad \varphi^{\prime}\left(0, \lambda_{n}\right)=h
\end{array}\right.
$$

and

$$
\left\{\begin{array}{l}
-\phi_{n}^{\prime \prime}(x)-C \phi_{n}(x)=\lambda_{n} \phi_{n}(x) \\
\phi_{n}(0)=1, \quad \phi_{n}^{\prime}(0)=h
\end{array}\right.
$$

The Sturm comparison theorem [17] says that the first positive zero of $\phi_{n}(x)$ is less than the first positive zero of $\varphi\left(x, \lambda_{n}\right)$. Let $h \neq \infty$. We have

$$
\phi_{n}(x)=\cos \left(\sqrt{\lambda_{n}+C} x\right)+\frac{h}{\sqrt{\lambda_{n}+C}} \sin \left(\sqrt{\lambda_{n}+C} x\right) .
$$

If $h=0$ then the first positive zero of $\phi_{n}(x)$ is

$$
\mu=\frac{\pi}{2 \sqrt{\lambda_{n}+C}} .
$$

If $h>0$, then the first positive zero of $\phi_{n}(x)$ is

$$
\mu>\frac{\pi}{2 \sqrt{\lambda_{n}+C}} \text {. }
$$

If $h<0$ and $\sqrt{\lambda_{n}+C}>|h|$, then the first positive zero of $\phi_{n}(x)$ is

$$
\mu>\frac{\pi}{4 \sqrt{\lambda_{n}+C}} .
$$

Let $h=\infty$, then the boundary conditions are $\varphi_{n}(0)=0, \quad \varphi_{n}^{\prime}(0)=1$, and

$$
\phi_{n}(x)=\frac{1}{\sqrt{\lambda_{n}+C}} \sin \left(\sqrt{\lambda_{n}+C} x\right),
$$

so the first positive zero of $\phi_{n}(x)$ in this case is

$$
\mu=\frac{\pi}{\sqrt{\lambda_{n}+C}} .
$$

In all cases $\nu$, defined by (44), is less than the first positive zero of $\varphi\left(x, \lambda_{n}\right)$. As the first positive zero of $\varphi\left(x, \lambda_{n}\right)$ is smaller than any other positive zero of $\varphi\left(x, \lambda_{j}\right), j=0, \cdots, n$, then, on the interval $(0, \nu)$ all $\varphi\left(x, \lambda_{j}\right), j=$ $0, \cdots, n$, do not change the sign, hence

$$
c_{j}=\frac{1}{\alpha_{j}^{2}} \int_{0}^{\pi} \chi_{\nu}(x) \varphi\left(x, \lambda_{j}\right) d x=\frac{1}{\alpha_{j}^{2}} \int_{0}^{\nu} \varphi\left(x, \lambda_{j}\right) d x \neq 0
$$

for $j=0, \cdots, n$.

Combining Lemmas $\mathbf{7 . 2}$ and $\mathbf{8 . 1}$, we see that if $q(x) \geq-C$ then the Mittag-Leffler series of $u^{x^{\alpha}}(0, t)$ (or $u_{x}^{x^{\alpha}}(0, t)$ if $\left.h=\infty\right)$ contains all $\lambda_{n} \geq N$, 
where $N$ is defined by either (33) or (34), and $Q$ is defined in Corollary $\mathbf{7 . 1}$, Denote

$$
\nu=\frac{\pi}{4 \sqrt{N+C}} .
$$

Then $u^{\chi_{\nu}}(0, t)$ (or $u_{x}^{\chi_{\nu}}(0, t)$ if $h=\infty$ ) carries all $\lambda_{n} \leq N$, so two initial conditions $x^{\alpha}$ and $\chi_{\nu}(x)$ are enough to recover the full boundary spectral data, and therefore, the diffusion coefficient $q$. We summarize the procedures of recovering $q$ in the following section.

\section{Algorithms}

We propose three algorithms for the cases, when $q \in L_{1}(0, \pi),\|q\|_{1} \leq Q$, and $q(x) \geq-C$, which reconstruct $q$ in finite steps.

\section{1. $q \in L_{1}(0, \pi)$, but no bound on $q$ is known a priori}

- Step 1. Start with an initial distribution $u(x, 0)=x^{\alpha}, \alpha \in(-1 / 2$, 0 ). Read $u(0, t)$ (if $h \neq \infty$ ), $u_{x}(0, t)$ (if $h=\infty$ ), $u(\pi, t)$ (if $H \neq$ $\infty$ ), $u_{x}(\pi, t)$ (if $H=\infty$ ) on $\left(T_{0}, T_{1}\right)$ and then extract the boundary spectral data $\left\{\lambda_{k_{j}}, \varphi\left(\pi, \lambda_{k_{j}}\right)\right\}$ if $H \neq \infty$ and $\left\{\lambda_{k_{j}}, \varphi^{\prime}\left(\pi, \lambda_{k_{j}}\right)\right\}$ if $H=$ $\infty$ by finding the poles and residues of the Laplace transform of the boundary reading. Lemma $\mathbf{7 . 1}$ guarantees that all $\lambda_{k}$ are recovered after a certain $N$.

- Step 2. Find the smallest $M$ such that starting from $M$ in each of the interval $\left(\left(k+a_{0}-1 / 2\right)^{2},\left(k+a_{0}+1 / 2\right)^{2}\right), \quad k \geq M$, there is exactly one eigenvalue recovered in Step 1 and none falls between them. Tentatively rank it as $\lambda_{k}$.

- Step 3. Count the number of eigenvalues obtained from Step 1 in the interval $\left(-\infty, \lambda_{M}\right)$, say $L$.

: If $L=M$ no missing eigenvalues. Proceed to Step 4. (One measurement only!)

: If $M-L>0$, then $M-L$ is the number of eigenvalues not recovered in Step 1. All the missing $M-L$ boundary spectral data can be now recovered by trying additional initial distributions

$$
f(x)=x^{j}, j=0,1, \cdots,
$$

one by one until all $M-L$ missing eigenvalues are extracted. This process stops in finite time. Rerank the eigenvalues if necessary.

- Step 4. From the full set of boundary spectral data obtain the full spectral data $\left\{\lambda_{k}, \alpha_{k}\right\}_{k \geq 0}$ by Lemma 6.1

- Step 5. Knowing the full spectral data $\left\{\lambda_{k}, \alpha_{k}\right\}_{k \geq 0}$ use Lemma 6.1 to recover $q$. 


\subsection{Suppose that we know a priori that $\|q\|_{1} \leq Q$}

Then we know explicitly the value of $N$, beyond which no single eigenvalue is missing by Lemma $\mathbf{7 . 2}$. Thus we use the above algorithm with the following modification

- Step $1^{\prime}=$ Step 1

- Step $2^{\prime}=$ Set $M=N$ which is given explicitly by either (33) or (34).

- Step $3^{\prime}=$ Modify step 3 as

$$
f(x)=x^{j}, j=0,1, \cdots, N .
$$

- Step $4^{\prime}=$ Step 4

- Step $5^{\prime}=$ Step 5 .

\subsection{Suppose we know that $q(x) \geq-C$, where $C>0$}

Then after Step 1, we use Corollary 7.1 to compute a upper bound $Q$ for $\|q\|_{1}$ and so $N$ is known explicitly. Although the ranking of eigenvalues after Step 1 may be false, formula in Corollary $\mathbf{7 . 1}$ with false numbering still gives the correct $\|q+C\|_{1}$. Lemma 9.1 says that only one more initial condition (43), where $\nu$ is defined by (46), is needed to recover all the missing eigenvalues. Thus we can reconstruct $q$ from just at most two observations.

- Step $1^{\prime \prime}=$ Step 1

- Step $2^{\prime \prime}=$ Compute $Q$ by Corollary [7.1, where recovered eigenvalues after Step $1^{\prime \prime}$ were given a tentative ranking. Compute $N$ by either (33) or (34).

- Step $3^{\prime \prime}=$ Use the step function in (43), where $\nu$ is defined by (46), as the second initial condition to extract all missing boundary spectral data.

- Step $4^{\prime \prime}=$ Step 4

- Step $5^{\prime \prime}=$ Step 5 .

\section{Uniqueness}

Now we show that these measurements determine $q$ uniquely. Consider the case $h, H \neq \infty$. The other cases can be proved similarly. We have

Lemma 11.1. Let $T>0,-\frac{1}{2}<\alpha<0$. For any $q \in L_{1}(0, \pi)$ there exists $N$ such that $q$ is uniquely determined by a finite number of measurements $u^{x^{\alpha}}(0, t), u^{x^{0}}(0, t), u^{x^{1}}(0, t), \cdots, u^{x^{N}}(0, t)$, and $u^{x^{\alpha}}(\pi, t), u^{x^{0}}(\pi, t)$, $u^{x^{1}}(\pi, t), \cdots, u^{x^{N}}(\pi, t)$, on an interval $\left(T_{0}, T_{1}\right)$. 
P r o o f. Suppose for any $N>0$ two diffusion coefficients $q, \tilde{q} \in$ $L_{1}(0, \pi)$ give the same measurements

$$
\begin{gathered}
u^{x^{\alpha}}(0, t), u^{x^{0}}(0, t), u^{x^{1}}(0, t), \cdots, u^{x^{N}}(0, t), u^{x^{\alpha}}(\pi, t), \\
u^{x^{0}}(\pi, t), u^{x^{1}}(\pi, t), \cdots, u^{x^{N}}(\pi, t), \quad t \in\left(T_{0}, T_{1}\right) .
\end{gathered}
$$

Let $\left\{\lambda_{n}\right\}$ and $\left\{\varphi\left(x, \lambda_{n}\right)\right\}$ be the set of eigenvalues, numbered in increasing order, and the set of associated normalized eigenfunctions $\left(\varphi\left(0, \lambda_{n}\right)=1\right)$, induced by $q$. Similarly, let $\left\{\tilde{\lambda}_{n}\right\}$ and $\left\{\tilde{\varphi}\left(x, \lambda_{n}\right)\right\}$ be the set of eigenvalues and the set of normalized eigenfunctions associated with $\tilde{q}$. Thus, any $f \in L_{2}(0, \pi)$ can be expanded into two eigenfunction expansions

$$
f(x)=\sum_{n \geq 0} c_{n} \varphi\left(x, \lambda_{n}\right)=\sum_{n \geq 0} \tilde{c}_{n} \tilde{\varphi}\left(x, \lambda_{n}\right) .
$$

The coefficient $q$ yields the diffusion distribution

$$
u(x, t)=\sum_{n \geq 0} c_{n} E_{\alpha}\left(-\lambda_{n} t^{\alpha}\right) \varphi\left(x, \lambda_{n}\right),
$$

with the diffusion at the end point $x=0$

$$
u(0, t)=\sum_{n \geq 0} c_{n} E_{\alpha}\left(-\lambda_{n} t^{\alpha}\right)
$$

The coefficient $\tilde{q}$ yields the diffusion distribution

$$
\tilde{u}(x, t)=\sum_{n \geq 0} \tilde{c}_{n} E_{\alpha}\left(-\tilde{\lambda}_{n} t^{\alpha}\right) \tilde{\varphi}\left(x, \lambda_{n}\right)
$$

with the diffusion at the end point $x=0$

$$
\tilde{u}(0, t)=\sum_{n \geq 0} \tilde{c}_{n} E_{\alpha}\left(-\tilde{\lambda}_{n} t^{\alpha}\right)
$$

Since $u(0, t)$ is analytic in $t>0$, the measurement of $u(0, t)$ on any finite time interval $\left(T_{0}, T_{1}\right)$ is sufficient to determine $u(0, t)$ uniquely for all $t>0$. Thus, $q$ and $\tilde{q}$ give the same boundary functions

$$
u^{x^{\alpha}}(0, t), u^{x^{0}}(0, t), u^{x^{1}}(0, t), \cdots, u^{x^{N}}(0, t), \quad t \in(0, \infty) .
$$

In other words,

$$
\sum_{n \geq 0} c_{n} E_{\alpha}\left(-\lambda_{n} t^{\alpha}\right)=\sum_{n \geq 0} \tilde{c}_{n} E_{\alpha}\left(-\tilde{\lambda}_{n} t^{\alpha}\right), \quad t>0 .
$$

The Laplace transforms of left and right hand sides of (48) are the same, and therefore, they have the same poles and residues. Hence, Lemma $\mathbf{5 . 1}$ tells us that (48) holds if, and only if, for any $n$ with $c_{n} \neq 0$ there exists unique $k$ such that $\lambda_{n}=\tilde{\lambda}_{k}$ and $c_{n}=\tilde{c}_{k}$, and for any $k$ with $\tilde{c}_{k} \neq 0$ there exists unique 
$n$ such that $\lambda_{n}=\tilde{\lambda}_{k}$ and $c_{n}=\tilde{c}_{k}$. So, the set $\left\{\lambda_{n} \mid c_{n} \neq 0\right\}$ and the set $\left\{\tilde{\lambda}_{n} \mid \tilde{c}_{n} \neq 0\right\}$ are the same. But the set $\left\{\lambda_{n} \mid c_{n}^{f} \neq 0\right.$ for at least one $f$ from $x^{\alpha}$, and $\left.x^{j}, j=0, \cdots, N\right\}$ coincides with the set of eigenvalues $\left\{\lambda_{n}\right\}_{n>0}$, and the set $\left\{\tilde{\lambda}_{n} \mid \tilde{c}_{n}^{f} \neq 0\right.$ for at least one $f$ from $x^{\alpha}$, and $\left.x^{j}, j=0, \cdots, N\right\}$ coincides with the set of eigenvalues $\left\{\tilde{\lambda}_{n}\right\}_{n \geq 0}$. Thus, $\left\{\lambda_{n}\right\}_{n \geq 0}=\left\{\tilde{\lambda}_{n}\right\}_{n \geq 0}$. Similarly, we can show that $\left\{\varphi\left(\pi, \lambda_{n}\right)\right\}_{n \geq 0}=\left\{\tilde{\varphi}\left(\pi, \lambda_{n}\right)\right\}_{n \geq 0}$, and hence $\left\{\alpha_{n}\right\}_{n \geq 0}=\left\{\tilde{\alpha}_{n}\right\}_{n \geq 0}$. But the diffusion coefficient $q$ is uniquely determined by the full spectral data $\left\{\lambda_{n}, \alpha_{n}\right\}_{n \geq 0}$. Thus $q=\tilde{q}$. Lemma 11.1 is proved.

We now summarize the above discussion into the following main result.

Theorem 11.1. Consider the fractional diffusion equation (11) where $q \in L_{1}(0, \pi)$. Then $q$ can be recovered uniquely from a finite number of readings (18) at $x=0$ and $x=\pi$ for $t \in\left(T_{0}, T_{1}\right)$. Furthermore, if a upper bound $Q$ of $\|q\|_{1}$ is known, then the number of readings $N$ is known explicitly as a function of $Q$ by (33) or (34). If a lower bound $q(x) \geq-C$ is known a priori then at most two measurements are enough to recover $q$ uniquely.

P r o o f. The steps in the previous algorithms contain the details of the initial conditions and the measurements to be used in each case.

\section{References}

[1] S.A. Avdonin, M.I. Belishev, and Yu. Rozhkov, The BC-method in the inverse problem for the heat equation. J. Inv. Ill-Posed Probl. 5 (1997), 309-322.

[2] S.A. Avdonin, S. Lenhart, and V. Protopopescu, Determining the potential in the Schrödinger equation from the Dirichlet to Neumann map by the boundary control method. Inverse problems: modeling and simulation. J. Inv. Ill-Posed Probl. 13 (2005), 317-330.

[3] M.I. Belishev, Canonical model of a dynamical system with boundary control in inverse problem for the heat equation (in Russian). Algebra i Analiz 7 (1995), 3-32; Transl. in: St. Petersburg Math. J. 7 (1996), 869-890.

[4] A. Boumenir and Vu Kim Tuan, An inverse problem for the heat equation. Proc. Amer. Math. Soc. 138, No 11 (2010), 3911-3921.

[5] A. Boumenir and $\mathrm{Vu}$ Kim Tuan, Recovery of a heat equation by four measurements at one end. Numer. Funct. Anal. and Optimiz. 31, No 2 (2010), 155-163. 
[6] A. Boumenir and Vu Kim Tuan, Inverse problems for multidimensional heat equations by measurements at a single point on the boundary. Numer. Funct. Anal. and Optimiz. 30, No 11-12 (2010), 1215-1230.

[7] A.V. Chechkin, R. Gorenflo, I.M. Sokolov, and V.Yu. Gonchar, Distributed order time-fractional diffusion equation. Fract. Calc. Appl. Anal. 6, No 2 (2003), 259-279.

[8] G. Freiling and V. Yurko, Inverse Sturm-Liouville Problems and their Applications. Nova Science Publishers, Inc., Huntington, New York, 2001.

[9] R. Gorenflo and F. Mainardi, Fractional calculus: Integral and differential equations of fractional order. In: A. Carpinteri and F. Mainardi (Eds): Fractals and Fractional Calculus in Continuum Mechanics. Springer Verlag, Wien and New York, 1997, 223-276.

[10] R. Gorenflo and F. Mainardi, Power laws, random walks, and fractional diffusion processes as well scaled refinement limits. In: A. Le Méhauté, J.A. Tenreiro Machado, J.C. Trigeassou and J. Sabatier (Eds): Fractional Differentiation and its Applications. U-Books, 2005, 497-516.

[11] R. Gorenflo, F. Mainardi, D. Moretti, G. Pagnini, and P. Paradisi, Fractional diffusion: probability distributions and random walk models. Physica A 305, No 1/2 (2002), 106-112.

[12] V. Isakov, On uniqueness in inverse problems for semilinear parabolic equations. Arch. Rational Mech. Anal. 124, No 1 (1993), 1-12.

[13] V. Isakov, Inverse Problems for Partial Differential Equations, 2nd Ed. Applied Mathematical Sciences 127, Springer, New York, 2006.

[14] A. Katchalov, Y. Kurylev, and M. Lassas, Inverse Boundary Spectral Problems. Monographs and Surveys in Pure and Applied Mathematics 123, Chapman \& Hall/CRC, Boca Raton, 2001.

[15] B.M. Levitan, Inverse Sturm-Liouville Problems. VNU Science Press, Utrecht, 1987.

[16] B.M. Levitan and M.G. Gasymov, Determination of a differential equation by two of its spectra. Russ. Math. Surveys 19 (1964), 1-62.

[17] B.M. Levitan and I.S. Sargsjan, Introduction to Spectral Theory: Selfadjoint Ordinary Differential Operators. Translations of Mathematical Monographs, Vol. 39, American Mathematical Society, Providence, R.I., 1975.

[18] F. Mainardi and R. Gorenflo, The Mittag-Leffler function in the Riemann-Liouville fractional calculus. In: A.A. Kilbas (Ed.): Boundary Value Problems, Special Functions and Fractional Calculus. Proceedings of the International Conference, Minsk, Feb. 16-21, 1996. Belorussian State University, 1996, 215-225. 
[19] V.A. Marčenko, Some questions in the theory of one-dimensional linear differential operators of the second order. American Mathematical Society Translations, Ser. 2. Vol. 101: Six Papers in Analysis, 1-104; American Mathematical Society, Providence, R.I., 1973.

[20] I. Podlubny, Fractional Differential Equations. An Introduction to Fractional Derivatives, Fractional Differential Equations, Some Methods of Their Solution and Some of Their Applications. Academic Press, San Diego-Boston-New York-London-Tokyo-Toronto, 1999.

[21] S.G. Samko, A.A. Kilbas, and O.I. Marichev, Fractional Integrals and Derivatives. Theory and Applications. Nauka i Teknika, Minsk, 1987 (In Russian); Translated by Gordon and Breach, Switzeland, 1993.

[22] E. Scalas, R. Gorenflo, and F. Mainardi, Fractional calculus and continuous-time finance. Physica A 284 (2000), 376-384.

Department of Mathematics

University of West Georgia

GA - 30118, USA

e-mail:vu@westga.edu

Received: July 31, 2010 